\title{
Endoreduplication Cycles during Hypocotyl Growth of Cabbage (Brassica oleracea L.) under Light and Dark Conditions
}

\author{
Nobuhiro $\mathrm{KUDO}^{\mathrm{I}}$ and Masahiro $\mathrm{MII}^{2}$ \\ 'Division of Plant Biotechnology, Gunma Agricultural Research Center, 493 Nishi-Obokata, \\ Sawa-Azuma, Gunma 379-2224, Japan \\ ${ }^{2}$ Faculty of Horticulture, Chiba University, 648 Matsudo, Chiba 271-8510, Japan \\ *Corresponding author E-mail address: kudo-n@pref.gunma.jp
}

Received 6 July 2004; accepted 23 August 2004 (Edited by Y. Hotta)

\begin{abstract}
Cabbage (Brassica oleracea L.) seedlings pursue two contrasting morphogenetic patterns, depending on the light environment. In the light, cabbage seedlings show short hypocotyls and open, unfolded cotyledons. In darkness, seedlings have markedly elongated hypocotyls and folded cotyledons. Durig hypocotyl growth, a majority of cells goes through endoreduplication. In light-grown hypocotyls, up to three cycles of endoreduplication occur, whereas in darkness about $10 \%$ of the total cells undergo the fourth cycles of endoreduplication. In both light and dark conditions, two cycles of endoreduplication take place prior to any significant hypocotyl growth. In darkness, the fourth cycle is completed very early during hypocotyl growth. These results support the view that endoreduplication may be a developmental programs in cabbage plants.
\end{abstract}

Key words: Brassica oleracea L., endoreduplication, flow cytometry, seedling development, skotomorphogenesis.

\section{Abbreviations}

\section{DAPI: 4', 6- diamidino-2-phenylindole}

Endoreduplication is a process of recurrent DNA duplication of the cell cycle without chromosomal and cellular division and leads to cells with multiple ploidy levels. Endoreduplication is very common in eukaryotes and it is estimated to occur in over $90 \%$ of angiosperms (Barlow, 1978; Nagl, 1978). In plants, endoreduplication is usually related to cell differentiation and growth (Kondorosi et al., 2000) and a clear relationship is observed between endoreduplication and cell size (Melaragno et al., 1993). Systemic endoreduplication has recently been described in Mesembryanthemum crystallinum (DeRocher et al., 1990), Arabidopsis thaliana (Galbraith et al., 1991), cucumber (Gilissen et al., 1993) tomato (Smulders et al., 1994), and radish (Kudo and Kimura, 2002).

Endoreduplication can be regulated by interactions between the environment cues and endogenous developmental programs. Plant growth conditions can influence patterns of endoployploidy. Leaves of tomato plants grown in a greenhouse exhibit higher levels of endopolyploidy than those of in vitro grown tomato plants (Sumlders et al., 1994). Similar ploidy variations have been described in potato (Uijtewaal, 1987). The extent of endoreduplication of maize endosperm is sensitive to water deficit (Artlip et al., 1995) and heat stress (Engelen-Eigles et al., 2000).

In cabbage, previous investigations have revealed that several rounds of endoreduplication occur in various organs and that distribution of the cells with different ploidy levels is organ-specific and developmental stage-specific (Kudo and Kimura, 2001a, b, c). In the present study, effect of light on the relative DNA content was examined by flow cytometry during hypocotyl growth.

In vitro plants of Brassica oleracea $\mathrm{L}$. cv. IroDori were grown from seeds and used in this study. Seeds were surface-sterilized as previously described by Kudo and Kimura (2001a). Two seeds were plated on a nutrient medium (Kudo and Kimura 2001a) in a 300-ml plastic bottle and kept in a growth chamber. Culture was maintained at $25^{\circ} \mathrm{C}$ under cool white fluorescent lights $(50 \mu \mathrm{mol}$ $\mathrm{m}^{-2} \mathrm{~s}^{-1}$ ) with a $16-\mathrm{h}$ day light / 8-h dark photoperiod or under dark conditions, in which the bottles were placed in opaque bags in the same growth chamber. 
Intact embryos and hypocotyls of germinating seedlings were subjected to flow cytometric analysis. Embryos were dissected from seeds with a fine dissecting needle. Nuclei were extracted from the embryos by chopping with a razor blade in $0.5 \mathrm{ml}$ of nuclei extraction buffer (solution A of PARTEC High Resolution Kit type P, Partec GmbH, Münster, Germany) according to the manufacturer's instructions. After filtration through a $30-\mu \mathrm{m}$ Cell Trics filter, $1.5-2.0 \mathrm{ml}$ of staining solution containing the dye DAPI (4', 6-diamidino -2-phenylindole, solution $\mathrm{B}$ of the kit) was added. The analyses were performed with a PAS flow cytometer (Partec) equipped with an HBO lamp for UV. For each sample, a minimum of 3000 nuclei (total count) were analyzed. Nuclear DNA content were measured with at least 4 replicates. To determine the standard peak position of $2 \mathrm{C}$ cells, the $2 \mathrm{C}$ peak from nuclei of in vitro grown young leaves was analyzed on each measurement. The data are plotted on a semi-logarithmic scale, so that the histogram peaks from $2 \mathrm{C}$ to $32 \mathrm{C}$ were evenly distributed along the abscissa. The data was presented as percentage of the total amount of nuclei in all peaks of the

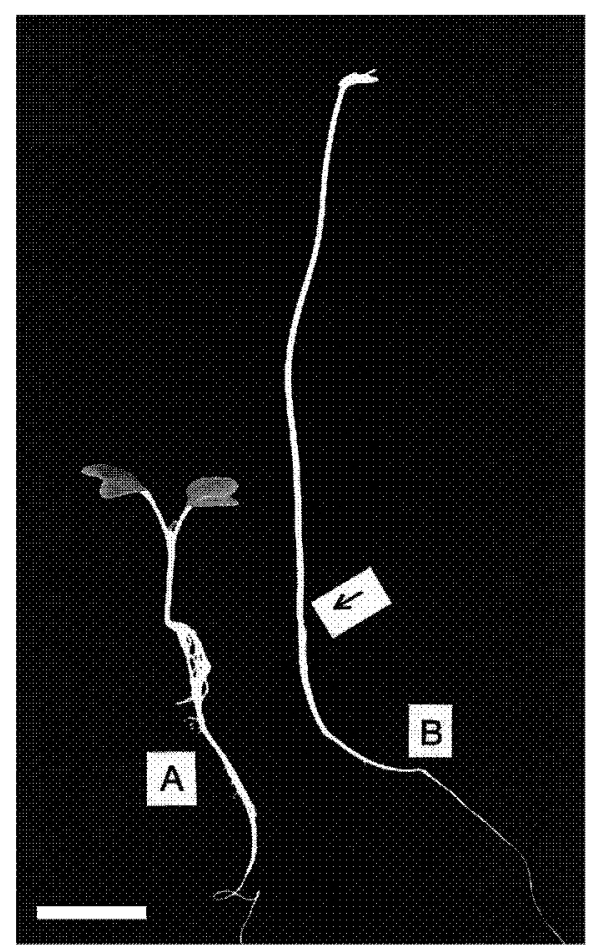

Fig. 1 Phenotypes of light-and dark-grown Brassica oleracea seedlings: light-grown seedling (A) and dark-grown seedling (B). In light, the seedlings exhibit short hypocotyls and unfolded cotyledons. In darkness, seedlings undergo etiolated growth or skotomorphogenesis. Darkgrown seedlings have an extremely elongated hypocotyl and folded cotyledons. Arrow indicates the start of roots. histogram.

The length of a whole hypocotyl was measured under a binocular microscope after spreading the seedlings on $0.8 \%$ agarose in a Petri dish. Each value is the average length of at least 10 individuals. The ploidy level and the hypocotyl length were followed for seedlings till 10 days after imbibition.

Depending on the light environment, cabbage seedlings pursued two contrasting morphogenetic patterns (Fig. 1). Light induced the photomorphogenetic programs; in light, the seedlings exhibit short hypocotyls and develped cotyledons. In darkness, seedlings underwent etiolated growth or skotomorphogenesis with an extremely elongated hypocotyl and folded cotyledons. An apical hook was formed and the reduction of the secondary root growth was observed. The dark-grown hypocotyl was almost six times as long as the light-grown hypocotyl (Fig. 2).

Fig. 3 shows the ploidy variation observed for 7 $\mathrm{d}$ old seedlings in light and darkness. Light-grown hypocotyls showed up to three rounds of endoreduplication, giving rise to $2 \mathrm{C}, 4 \mathrm{C}, 8 \mathrm{C}$ and $16 \mathrm{C}$ nuclei. Light-grown hypocotyls gave four histograms, where $4 \mathrm{C}$ and $8 \mathrm{C}$ peaks were prevalent and the proportion of the $16 \mathrm{C}$ nuclei was small (Fig. 3A). In

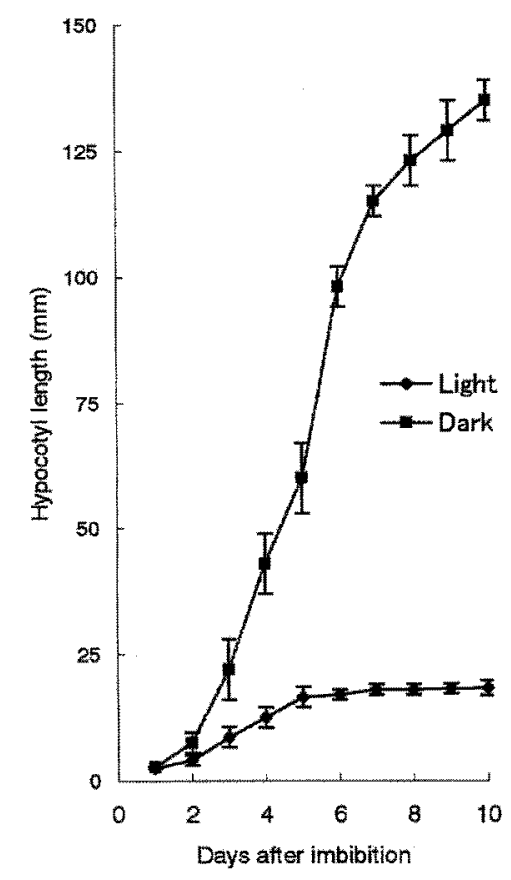

Fig. 2 Hypocotyl growth of seedlings in light and in darkness. At day 2, dark- grown hypocotyls are slightly longer than those grown in light. Between day 2 and day 5 , light-grown hypocotyls show an exponential growth whereas in darkness hypocotyls exhibits an exponential growth between day 3 and day 7. Dark-grown hypocotyls are almost six times longer than those grown in light at day 5 . 

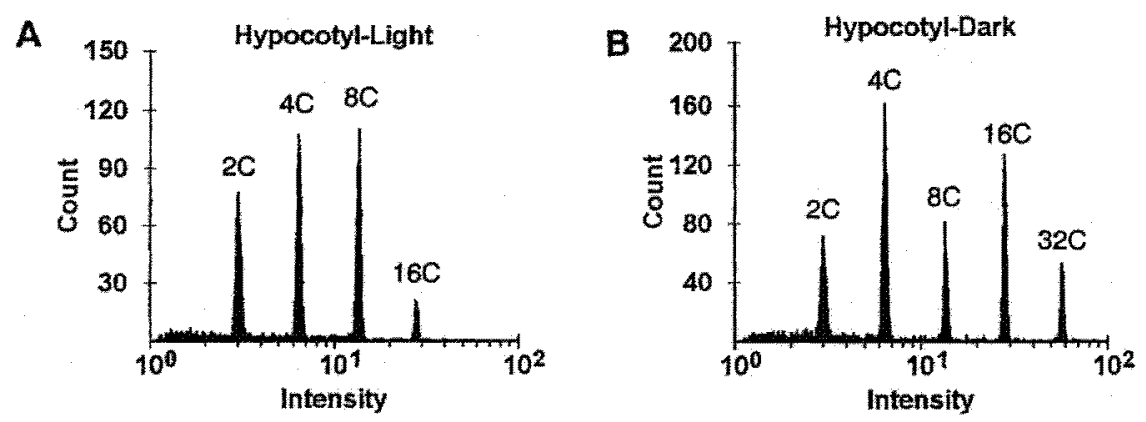

Fig. 3 Characteristic histograms of nuclei distribution of Brassica oleracea hypocotyls grown in light (A) and in darkness (B). Light-grown hypocotyl show four ploidy levels (2C16C). Etiolated hypocotyl shows cells of five ploidy levels $(2 \mathrm{C}-32 \mathrm{C})$.

etiolated seedlings, elongated hypocotyl tissues contained cells with five ploidy levels $(2 \mathrm{C}, 4 \mathrm{C}, 8 \mathrm{C}$, $16 \mathrm{C}$ and $32 \mathrm{C}$ ), and a distinct and reproducible peak for $32 \mathrm{C}$ nuclei appeared (Fig. 3B), at about $10 \%$ of all nuclei detected in hypocotyl extracts. Consequently, the fourth cycle of DNA replication in hypocotyls was specific for etiolated development.

Fig. 4 shows the evolution of the ploidy level during hypocotyl development. In the mature embryos of dry seeds (day 0 ), only $2 \mathrm{C}$ nuclei were detected. The samples taken at day 0 contained nuclei from whole embryos because they were too small to be divided into different parts. Already on day 1, DNA synthesis and subsequent endoreduplication had taken place under both growth conditions as indicated by two peaks of $4 \mathrm{C}$ and $8 \mathrm{C}$ DNA levels, accounting for $55 \%(4 \mathrm{C})$ and $22-28 \%(8 \mathrm{C})$ of total nuclei, respectively. There were no difference in the ploidy level between the seedlings grown in the light and in the darkness on day 1 . Since no significant hypocotyl elongation had occurred at this stage in eihter growth conditions as shown in Fig. 2, endoreduplication is considered to precede hypocotyl growth. Because cabbage is a diploid species $(2 n=18)$, the $4 \mathrm{C}$ DNA level represents a $\mathrm{G} 2$ phase of mitotic cycle or a multiploid form of the genome in endoreduplication cycle. The presence of $8 \mathrm{C}$ nuclei is an indicator of the capacity of cells to enter endoreduplicatoin cycles. At day 2, dark-grown hypocotyls were slightly longer than those grown in light. The third round of endoreduplication was detected under both growth conditions, although the proportion of $16 \mathrm{C}$ nuclei in darkness was higher than that in light $(16.8 \%$ vs. $4.6 \%)$.

Between day 2 and day 5, light-grown hypocotyls went through an exponential growth phase (Fig. 2). During this period, the percentage of $4 \mathrm{C}$ and $8 \mathrm{C}$ nuclei had slightly reduced, with a concomitant increase in $2 \mathrm{C}$ peak (Fig. 3). Between day 5 and day 10, hypocotyl growth came to an end (Fig.
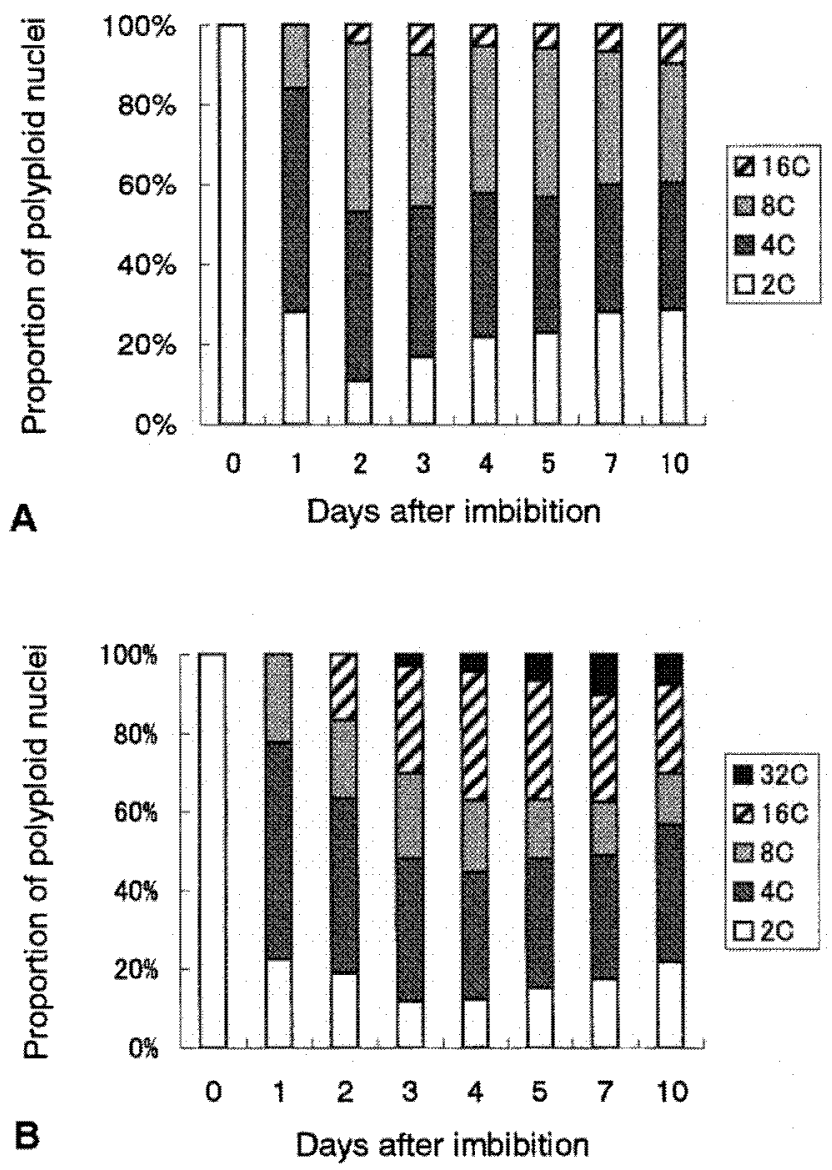

Fig. 4 Evolution of ploidy levels in hypocotyls during seedling development: (A) Ploidy patterns of hypocotyls from light-grown seedlings, (B) ploidy patterns of hypocotyls from dark-grown seedlings. $32 \mathrm{C}$ nuclei appeared very early during hypocotyl development (day 3 ) in darkness

2) and an increase in the proportion of $2 \mathrm{C}$ and $16 \mathrm{C}$ nuclei occurred.

In darkness, between day 3 and day 7, hypocotyls showed an exponential growth with typical morphological sign of etiolated growth (Fig. 2). At day 3, a small but reproducible $32 \mathrm{C}$ peak $(1-2 \%)$ was present, indicating that the fourth round of endoreduplication had taken place (Fig. 4B). Between day 3 and day 7 , the proportion of $2 \mathrm{C}$ and $32 \mathrm{C}$ nuclei 
increased. Between day 7 and day 10, elongation of hypocotyl still continued. During this period, a slight decrease of the $32 \mathrm{C}$ peak were detected.

Previous studies showed that somatic cells in Brassica seedlings go through several rounds of endoreduplication and that this process is developmentally regulated (Kudo and Kimura, 2001a, b, c). Brassica embryos apparently contain cells that may be programmed to enter endoreduplication cycles upon seedling development. In the present study, this phenomenon was examined further, in particular to address a question as to whether environmental cues influence endoreduplication levels or not. Data presented here show that (i) in both light and dark growth conditions endoreduplication preceded growth of hypocotyls; (ii) in dark-grown hypocotyls $32 \mathrm{C}$ nuclei appeared early during hypocotyl growth (day 3) and that the fourth round of endoreduplication cycle is specific to dark growth conditions. The patterns of endoreduplication in light - and dark-grown hypocotyls support the view that the number of endoreduplication cycle may be affected by light conditions. Similar results has previously been reported in dark-grown epicotyls of Pisum sativum which showed higher ploidy levels than those grown in light (van Oosteveldt and van Parijs, 1975). Environmental cues such as light can be involved in signal transduction of the endoreduplication process (Traas et al.,1998), although no clear view exists on how environmental cues modulate the endoreduplication process. More factors are required to identify in order to understand the regulation and the function of endoreduplication.

\section{References}

Artlip, T.S., Madison, J.T., Setter, T.L., 1995. Water deficit in developing endosperm of maize: cell division and nuclear DNA endoreduplication. Plant Cell Environ., 18: $1034-1040$.

Barlow, P.W., 1978. Endopolyploidy: towards an understanding of its biological significance. Acta Biotheor., 27: $1-18$.

DeRocher, E.J., Harkins, K.R,, Galbraith, D.W., Bohnert, H. J., 1990. Developmentally regulated systemic endopo- lyploidy in succulents with small genomes. Science, 250: $99-101$.

Engelen-Eigles, G., Jones, R.J., Phillips, R.L., 2000. DNA endoreduplication in maize endosperm cells: the effect of exposure to short-term temperature. Plant Cell Environ., 23: $657-663$.

Galbraith, D.W., Harkins, K.R., Knapp, S., 1991. Systemic endopolyploidy in Arabidopsis thaliana. Plant Physiol., 96: $985-989$.

Gilissen, L.J. W., Van Staveren, M.J., Hakkert, J.C., Creemers-Molenaar, J., Verhoeven, H. A., 1993. Development of polysomaty in seedlings and plants of Cucumis sativus L. Plant Sci., 91: 171-179.

Kondorosi, E, Roudier, F., Gendreau, E., 2000. Plant cellsize control: growing by ploidy? Curr. Opin. Plant Biol., 3: 488- 492 .

Kudo, N., Kimura, Y., 2001a. Flow cytometric evidence for endopolyploidy in seedlings of some Brassica species. Theor. Appl. Genet., 102: 104-110.

Kudo, N., Kimura, Y., 2001b. Patterns of endopolyploidy during seedling development in cabbage (Brassica oleracea L.). Ann. Bot., 87: 275- 281.

Kudo, N, Kimura, Y., 2001c. Flow cytometric evidence for endopolyploidization in cabbage (Brassica oleracea L.) flowers. Sex. Plant Rep., 13: 279-283

Kudo, N., Kimura, Y., 2002. Flow cytometric analysis for systemic endopolyploidy in development of radish (Raphanus sativus L.). Plant Biotechnol., 19: 45-52.

Nagl, W., 1978. Endopolyploidy and polyteny in differentiation and evolution. Elsevier, Amsterdam.

Melaragno, J.E., Mehrotra, B., Coleman, A.W., 1993. Relationship between endopolyploidy and cell size in epidermal tissue of Arabidopsis. Plant Cell, 5: 1661-1668.

Smulders, M.J.M., Rus-Kortekaas, W., Gilissen, L.J.M., 1994. Development of polysomaty during differentiation in diploid and tetraploid tomato (Lycopersicon esculentum) plants. Plant Sci., 97: 53-60.

Traas, J., Hülskamp, M., Gendreau, E., Höfte, H., 1998. Endoreduplication and development: rule without dividing? Curr. Opin. Plant Biol, 1: 498-503.

Uijtewaal, B.A., 1987. Ploidy variability in greenhouse cultured and in vitro propagated potato (Solanum tuberosum) monohaploids $(2 \mathrm{n}=\mathrm{x}=12)$ as determined by flow cytometry. Plant Cell Rep., 6: 252-255.

van Oosteveldt, P., van Parijs, R., 1975. Effect of light on nucleic acid synthesis and polyploidy level in elongating epicotyl cells of Pisum sativum. Planta, 124: $287-295$. 